\title{
Mapping Espoused Organizational Values
}

\author{
Humphrey Bourne ${ }^{1} \cdot$ Mark Jenkins $^{2} \cdot$ Emma Parry $^{2}$
}

Received: 23 May 2017 / Accepted: 6 November 2017 / Published online: 17 November 2017

(c) The Author(s) 2017. This article is an open access publication

\begin{abstract}
This paper develops an inventory and conceptual map of espoused organizational values. We suggest that espoused values are fundamentally different to other value forms as they are collective value statements that need to coexist as a basis for organizational activity and performance. The inventory is built from an analysis of 3112 value items espoused by 554 organizations in the UK and USA in both profit and not-for-profit sectors. We distil these value items into 85 espoused value labels, and these are assessed in terms of their similarity and difference through judgements made by 53 experienced individuals. The resulting conceptual map facilitates the evaluation of values which are espoused at the organizational level, as opposed to aggregations of personal values, an important distinction that is often ignored in the literature. This analysis identifies a number of distinct areas of emphasis occupied by espoused values. In particular, the richness of value labels that relates to broader ethical issues may be aimed at external stakeholder management, but also may have an increasing influence on organizational behaviour as they are embedded into organizational practices. By advancing our understanding of espoused values, through an analysis of those being used in practice, we provide a means by which future research into organizational values and ethical issues can progress.
\end{abstract}

Keywords Corporate values · Espoused values · Inventory · Multidimensional scaling organizational behaviour · Organizational values

\section{Introduction}

The values construct has been widely promoted as a basis for understanding the priorities and underpinning beliefs of organizations (Rokeach 1968). In particular, espoused values have been positioned as a critical element in the way that organizations deal with the need for cohesion and performance (Kabanoff et al. 1995) and as a basis for understanding the way in which ethical business issues are considered and addressed (Pruzan 2001). Espoused values are those 'determined by the top management team ... often

Humphrey Bourne

Humphrey.bourne@bristol.ac.uk

Mark Jenkins

Mark.jenkins@cranfield.ac.uk

Emma Parry

Emma.parry@cranfield.ac.uk

1 School of Economics, Finance and Management, University of Bristol, Priory Road Complex, Bristol BS8 1TN, UK

2 School of Management, Cranfield University, Cranfield, Bedfordshire MK43 0AL, UK stated explicitly in corporate documents' (Bansal 2003, p. 520). Over the past two decades, an increasing number of organizations, both profit and not-for-profit, have explicitly espoused their values on their websites. In a study of values relevant to business research, McDonald and Gandz (1991) reported that just three of the 32 organizations investigated were able to provide the authors with any documented list of values. Some 25 years later, almost eight out of ten US and UK organizations devoted web space to communicating their espoused values. Espoused values are distinct from other forms of organizational values including those that may be enacted (Howell et al. 2012), attributed to the organization, or shared amongst members (Bourne and Jenkins 2013). Recently, espoused values have been linked to organizational performance (Jonsen et al. 2015), social integration (Grøgaard and Colman 2016) and organizational commitment (Howell et al. 2012). At the same time, espoused values are acknowledged to involve a degree of impression management and sensitivity to cultural norms (Kabanoff and Daly 2002; Zander et al. 2016).

The impact of espoused values on organizational actions and their recent prominence on organization websites 
suggests that they should be a matter of academic interest and that it is timely to explore the phenomenon. The purpose of this paper is threefold: to construct an inventory of espoused values that captures the actual terms used by organizations on their websites; to explore relations between these value categories in order to build a conceptual map of espoused values; and to consider the ethical implications of this for organizing and organizations. We present an extensive inventory of espoused values, built from the words and phrases that are explicitly used by profit and not-for-profit organizations in the UK and the USA. The inventory, together with its synonyms, is grounded in the contemporary lexicon of a range of organizations and so is sufficiently comprehensive to capture value terms relevant to a variety of contexts. We then present an underlying structure of espoused values developed empirically through the aggregated judgements of 53 individuals working in a range of organizations by applying non-metric multidimensional scaling (MDS). The resulting map reveals a structural arrangement of espoused values that allows us to compare this with other structural arrangements in the organizational and individual values literature, and to consider the wider implications for ethical practices in organizations.

The paper is organized as follows. First, we review the literature on organizational values with a specific emphasis on espoused values and summarize the principal empirical studies that have contributed to our understanding. From there, we position the study of espoused values in the context of the wider work on organizational values and their structure. After an outline of our research design, we develop an approach that integrates a comprehensive inventory into a map of espoused organizational values, which we discuss in relation to earlier work. We go on to consider how such a framework can help our understanding of ethical practices in organizations and finally, we consider the limitations of this work and suggest further research that will build upon it.

\section{Organizational Values}

Organizational values have been positioned as central to concepts such as culture (Gagliardi 1986; Schein 1985) and organizational identity (Gioia et al. 2000; Scott and Lane 2000), and have been linked with other fields of organizational research including strategy (Bansal 2003; Carlisle and Baden-Fuller 2004), change (Burnes and Jackson 2011; Kabanoff et al. 1995) and leadership (Cha and Edmondson 2006; Stadler and Hinterhuber 2005). Comparing and contrasting the values of individuals and their organization has advanced understanding of the problems and challenges relating to person-organization fit (Cable and Edwards 2004; Kristof 1996), commitment (Finegan 2000; Stride and Higgs 2013) and employee attitudes (Ostroff et al. 2005). Organization values are associated with corporate governance
(Kabanoff and Nesbit 1997; Nohria and Ghoshal 1994), relationships with external constituents (Voss et al. 2000), ethical behaviour (Auster and Freeman 2013; Pruzan 2001) and firm performance (Jonsen et al. 2015). In short, organizational values are associated with a wide range of organizational phenomena, actions, orientations and outcomes.

Organizational values are different from, but related to, individual, cultural and societal values. Schwartz (1992) showed that the values of individuals arise out of psychological and social needs and are structured such that types of values are more or less compatible with one another, with the implication that individuals endorse certain values and reject those that are essentially opposite in nature. Cultural distinctions in values are revealed through the aggregation of the value priorities of individuals within the culture (Rokeach 1973; Schwartz 1992), while societal values are shaped by those in power (d'Andrade 2008) and represent that society's concerns and beliefs with regard to the effective functioning of its institutions (Fischer et al. 2010).

The first values of an organization are typically initiated by and reflect the individual values of the founders (Schein 1985). These initial values are sustained by organizational members (Zander et al. 2016), become embedded and reinforced through organizational structures and practices (Buenger et al. 1996) and modified from time to time in response to external or internal change (Amis et al. 2002). Organizational values, generally, include forms that reflect individual values of founders, aggregated 'shared' values of groups of members, those attributed to the organization, embedded in structures and processes, and those that represent collective concerns and beliefs with regard to its effective functioning, sanctioned and espoused by senior managers (Bourne and Jenkins 2013).

Espoused values therefore have a significant role in representing the intent of organizations to operate in particular ways and to encourage particular behaviours from organizational members. In this sense, they are aligned to the concept of business codes which represent the various codes of ethics, codes of practice and corporate ethics statements made by organizations which may impact ethical behaviour and performance (Kaptein and Schwartz 2008; Scholtens and Dam 2007). In recent years, espoused values have been linked directly to a number of organizational outcomes. Jonsen et al. (2015) suggest that espousing differential values improved financial performance through impression management, employee fit and clarity in focus. Meanwhile, Grøgaard and Colman (2016) found that despite-or perhaps because of-widespread local interpretation of the meaning and significance of espoused values amongst a MNE's subsidiaries, they had a positive impact on social integration. Similarly, Howell et al. (2012) found affective commitment to an organization increased when members perceived congruence between its espoused and enacted values. 
Conversely, however, they found affective commitment decreased when espoused values were not widely shared.

This last point brings to attention the extent to which espoused values can be viewed as accurate representations of prioritized values in organizations. Kabanoff and Daly (2002, p. 90) allude to this in their description of espoused values as those that 'reflect what senior managers actually believe their organization to be like, what they would like or prefer their organizations to be like, or what they would like significant stakeholders to believe the organization is like'. This description acknowledges the opportunity for impression management and conformity to social expectations. Because of the public nature of espoused values, senior managers are likely to be sensitive to cultural norms so emphasize more socially desirable values, exclude those that are undesirable and over-claim the most desirable and under-claim the least (Crane 1999; Randall and Fernandes 1991). Espoused values, therefore, may be somewhat sanitized both in what they include and in what is left out and so can only be an incomplete representation of the values of the organization (Zander et al. 2016). However, their partiality is an important organizational phenomenon; what is included and omitted is an indication of the way in which senior managers intend their organization both to operate and to be seen to be operating.

Regardless of the underlying intentions of espousing values, their presence on official websites will have an impact on the way that internal and external stakeholders perceive the organization. Making such values explicit allows obvious contradictions between espoused values and the actions of organizations to be exposed, while employees, customers and other stakeholders may self-select on the basis of their perceived congruence with the values (Grøgaard and Colman 2016; Jonsen et al. 2015). The case of US Energy conglomerate Enron, which filed for bankruptcy in 2002, was a notorious example of how the espoused values of an organization (in Enron's case these were Respect, Integrity, Communications and Excellence) were completely at odds with the values enacted by the organization in its daily operations (Kunen 2002; Lencioni 2002).

Espoused values are manifestations of senior managers' concerns for the effective functioning of their organization, and, as such, they are required to accommodate the needs and expectations of both internal and external stakeholders, as well as the context and the aims of the organization (Fischer et al. 2010). In doing so, the espoused values of any organization are likely to include combinations that compete in the sense that they would not typically sit together in the value systems of individuals (Schwartz 1992). Instead, these reveal the paradoxes that organizations are typically required to accommodate (Smith and Lewis 2011; Zander et al. 2016). Theoretical ideas of the structure of values at the level of the individual emphasize polarities (Rokeach
1973; Schwartz 1992), a preference for one value as opposed to another. The 'structure' of espoused values, however, is unlikely to reveal tensions within a value system as organizations may need simultaneously to include competing values in order to accommodate the various functions that they are required to undertake. Espoused values statements may combine what Quinn and Rohrbaugh (1983) describe as 'competing values' that are required to coexist within a single organization.

Espoused values are a distinct form of organizational values that are increasingly documented, and which are associated with organizational outcomes. The espousing of values may involve a degree of impression management and social conformity, so raising ethical questions, while structures of values reveal the paradoxes that organizations as complex structures need to accommodate. A rigorous basis for understanding and evaluating espoused values in organizations is therefore timely and can provide us with an important insight into the value items which are deliberately selected and promoted to characterize an organization. The recent trend for organizations to explicitly provide a statement of espoused values now affords us the opportunity to consider this aspect of organizations from a wider empirical perspective.

\section{Researching Organizational Values}

Rather than reflecting the way in which individuals describe the values of organizations as they see them, espoused values are constructed by multiple (often more senior) individuals as formal and deliberate representations of an organization's values. To date there has been no systematic empirical exploration of the espoused values of organizations to enable a comprehensive inventory and framework to be defined. In fact, the identification of the form of organizational values being investigated has been somewhat confused in earlier studies. Few scholars have put together scales to measure a particular form of organizational value, whereas some others use measures indiscriminately across value forms without recognizing that a framework designed to measure one value form might not be appropriate for another.

Inventories that have been adopted in a range of studies include the Organizational Cultural Profile (OCP) and McDonald and Gandz's (1991) list of business values, while researchers such as Jonsen et al. (2015) have generated inventories that are specific to the context they are studying. Inventories are typically derived from a combination of literature sources, interview data and document search and thus might not distinguish between the types of values measured. Prominent examples of frameworks of organizational values include the Competing Values Framework (Quinn and Rohrbaugh 1983), Kabanoff et al.'s (1995) Justice-Based Typology of Organizational 
Value Structures and Wiener's (1988) Types of Values framework. Perhaps the best-known framework of organizational values is Quinn and Rohrbaugh's (1983) Competing Values Framework. This features two primary competing dimensions: organizational focus (internal vs. external) and preferred structure (stability and control vs. flexibility and change). These dimensions form a typology comprising four types of organizational effectiveness: human relations; open systems; internal process; and rational goal. Each type encapsulates a set of values, although Quinn and Rohrbaugh did not elaborate these; instead, it has fallen to others to develop suitable measures of organizational values (Kalliath et al. 1999; Ostroff et al. 2005).

One of the problems with the aforementioned frameworks is the absence of any consideration or emphasis on the explicit, espoused values of the organization. One exception to this is the approach taken by Kabanoff et al. (1995) and Kabanoff and Daly (2002) who do explore the espoused values of organizations. Taking a distributive justice perspective, Kabanoff et al. focused on the competing demands of organizational cohesion which, they argued, favours 'equality' values, and organizational performance, which favours 'equity' values. Kabanoff and his colleagues' interest centred on distributive justice and the tensions between equality and equity, and consequently their inventory and typology each reflect these internal concerns. They do not set out to capture values that reflect organizations' relationships with their wider environment, which means that the application of their typology of espoused values is limited to studies with a similar internal focus.

In conclusion, we suggest that to advance research in organizational values an appropriate start point is the development of a comprehensive inventory of espoused organizational values derived from a wide empirical base. We also suggest that the opportunity for theory development will be advanced through a better understanding of the structural relationship between espoused values. In order to achieve this, we present an inventory derived from the values espoused by a large number of organizations in both the UK and the USA, which is then structured into a conceptual map through an empirical process to identify distance between value items.

\section{Methods}

The development of the conceptual map followed three distinct stages: first, the generation of a comprehensive list of organizational values via analysis of organizational websites; second, the use of card sort methodology to examine judgments of the associations between values; and third, the use of MDS to analyse the results of the card sort to generate a spatial model of organizational values from which a conceptual map can be developed.

\section{Generating an Inventory of Espoused Organizational Values}

We identified those words and phrases that are clearly intended by organizations to represent their values, via an examination of the websites of UK and US organizations. The UK sample comprised a total of 200 UK organizations from profit and not-for-profit (including the public and charity) sectors. Organizations from both sectors were included in order to promote maximum variation in the values espoused in support of our aim to develop as comprehensive an inventory as possible. Lists were obtained from: first, the Financial Times Stock Exchange (FTSE) 100 list of (private sector) companies (October 2013); second, the top 50 charities as measured by income by the Charity Commission for England and Wales (also October 2013); and third, the 50 largest English local government organizations as measured by population in the 2011 census. Local government organizations in England were selected because they represent a form of public sector organization common to many nations. Overall, 74\% of UK organizations displayed their values on their website, meaning that 52 of the 200 organizations ( 24 private sector, 16 charities, 12 local government) did not display their values on their websites. The US sample comprised the entire list of the 2013 Fortune 500 (Fortune.com 2013). Just over $80 \%$ of US organizations recorded their values prominently on their corporate websites, meaning that 98 of the Fortune 500 did not provide any explicit statement of values and were discarded. The UK set comprised the espoused values of 152 organizations, while the USA set comprised those from 402 organizations, so together a total of 554 organizations' statements of espoused values were recorded.

\section{Identifying Value Statements}

The corporate website of each organization was searched for a statement of values. The standard approach we used was to enter 'our values' into the search box of the website front page, which would in the majority of cases find the relevant page. When it did not, we would first search web pages that provided corporate information, often titled 'about us', then explore employment pages and finally pages devoted to corporate social responsibility. If none of these yielded a statement of values and after all reasonable alternatives had been exhausted (including the use of alternative terms, such as 'our commitments' or 'our ethos'), we concluded that any values the organization might espouse were not displayed in their web pages and recorded 'none found'. 
These represented $26 \%$ of UK organizations and $20 \%$ of US organizations.

Statements of values varied. Some organizations simply listed a number of value items without any elaboration, but most usually gave headline statements in the form of single words, such as 'passion', 'innovation', 'integrity', 'teamwork' and 'excellence' (Schroders 2014) and were followed with a short explanation below. On other websites, such as that of the UK food retailer J. Sainsbury, the values statements were presented as short phrases, typically followed by a short description: 'Best for food and health', 'Sourcing with integrity', 'Respect for our environment', 'Making a positive difference to our community' and 'A great place to work' (J. Sainsbury 2014). A smaller number of organizations expressed values in longer, less precise terms, as in 'Commitment to all our employees to treat them with respect and provide satisfying career opportunities' (Caesar's Entertainment 2014) or 'We believe that ethical and moral standards are the foundation of good business policies and will operate with integrity' (Huntsman 2014).

The decision was made to focus on the headline values statement and not to attempt to interpret any additional descriptions that may have been provided. We accept that excluding descriptions may in some individual instances mean overlooking a particular interpretation of a word or phrase. However, we consider that differences in the extent of elaboration, which range from none at all through short, single sentence descriptions to highly elaborate explanations, including the use of video and other media, meant that for the sake of consistency the level of analysis should be standard. Several organizations included double-, trebleand even quadruple-barrelled value statements. On many occasions these appeared linked, as in 'honesty and integrity' and 'teamwork and collaboration', but at other times the links were less clear, as in 'collaboration and growth' or 'have fun, work hard and execute'. While at times it was possible to code conjoined terms just once, at other times it was clearly not, so we took the decision to code separately each value contained in multiple value statements.

The UK organizations yielded a total of 744 value items, an average of just under five recorded for each organization. The US sample yielded a total of 2368 value items, an average of just under six for each organization. A total number of 3112 value items were therefore recorded for categorization, an overall average of 5.6 value items per organization. Developing the inventory from these value items was emergent and iterative, involving a number of rounds in which value labels were refined and extended as more value items were considered. Each of the three coders took the list of values elicited from organizations' web pages and separately assigned common value labels to each value item. The process was essentially emergent in that labels were created during the coding process, supported by the use of Chambers
Dictionary and Roget's Thesaurus, requiring some iteration to ensure internal consistency of each coder's categorization. During the initial coding, value terms that are synonyms of one another were grouped together. So, for example, a value label such as 'teamwork' might appear as 'act as a global team', 'we work in teams', 'working together' or simply 'one team'. Another value label of 'excellence' might variously be expressed by organizations as 'excellent performance', 'delivering excellence' or 'commitment to excellence'. The phrase 'we value....' was used by the coders, so that each value was stated as a noun (sometimes preceded by an active verb or adjective), to help provide a consistent terminology for the values statements they elicited.

All values were assigned to value labels independently by three different coders. Value items were recorded for each organization on a spreadsheet, using a separate cell for each value to allow for later manipulation. Once each coder had carried out this process independently, the lists of value labels were compared and any differences resolved via a discussion between the coders. As the inventory developed, the coders were consistently reaching agreement levels of 0.85 in the first round of coding. After both samples had been coded, a total of 85 value labels were identified.

\section{Mapping the Value Labels}

The association between the different values was examined via an online card sort exercise using OptimalSort software. Card sort is a technique used to organize information as it helps to establish the mental models that individuals use when considering particular data. In this case, card sort was used in order to obtain an assessment of the similarity or difference between the different values identified and to organize them into groupings.

The sample was self-selected from part-time students on executive MBA and Masters programmes at two UK Universities. Students on these programmes had a number of attributes that were considered favourable for the purpose of the research: they had a minimum of 5 years' experience of working in organizations; they came from across the profit and not-for-profit sectors; they represented a mix of gender, nationality and familiarity with the English language; and they were familiar with the academic and practical language of business and organizations. Respondents were invited to undertake the card sort via an email in which they were provided with a web-link to the card sort exercise on OptimalSort. Each respondent was asked to group the value items in ways that made sense to them. A free sorting approach was used (Giguère 2007) whereby respondents were not constrained a priori by the groups that they could use. However, respondents were provided with the guideline to place the list of 85 value labels into between 15 and 25 groupings. The software made it simple to move the value 
labels around from one grouping to another, to create new groupings or to remove others in the same way as would be the case if physical cards were used. A total of 53 card sorts were completed and used to generate a similarity matrix using the software. This provides the percentage of occasions that each value was paired with the other value, so a high percentage indicates that two are linked together in the same grouping for a high proportion of the time, while others may only link together occasionally or not at all. For example, the value label 'care for the environment' was associated with 'sustainability' by $73 \%$ of respondents but none associated it with 'tenacity' or 'confidence'. The similarity matrix therefore shows the relationship of every one of the 85 values to every other value. Those that were grouped together more frequently are considered by those who took part in the card sort, collectively, to have a greater similarity to one another than those that are grouped together infrequently or not at all.

\section{Multidimensional Scaling (MDS)}

As noted by Fincher and Tenenberg (2005), the challenge in using card sort is in making sense of the data. In order to achieve this, the similarity matrix was analysed using MDS (Borg and Groenen 2005). MDS is designed to convert proximities, such as those in the similarity matrix, into a geometric configuration or map of points in low-dimensional space (Kruskal 1964; Whaley and Longoria 2009). In the case of card sort, it allows us to examine the underlying dimensions represented by respondents' judgments without relying on the researchers' preconceptions (Rosenberg and Kim 1975).

In order to prepare the data for MDS, the data in the similarity matrix were converted from percentages to proportions by dividing each cell by 100 so that the relationship between each value label was expressed as a value between 0 (no relationship) and 1 (complete relationship). The data were imported into SPSS, and the MDS programme PROXSCAL was executed. In the first instance, normalized stress scores were generated for between one and six dimensions in order to determine the most appropriate number of dimensions. Stress scores measure goodness-of-fit and are affected by factors including the number of points in the data and the dimensionality of the space; normalization of stress scores removes scale dependency (Borg and Groenen 2005). Broadly, as the number of dimensions is raised, the stress scores lower logarithmically until there is no discernible improvement in the score. Borg and Groenen suggest that, as a general rule, the stress score should be below 0.15 for there to be confidence in the resulting scaling, but they also point out that this is indicative only and that reasons for accepting a somewhat higher stress score include ease of interpretation. Clearly, a two-dimensional model would present more easily interpretable solutions than would a three-dimensional model as each additional dimension greatly reduces the clarity of the resulting graphical displays. In his seminal work on values, Schwartz (1992) elected to use two-dimensional plots for this reason in developing spatial relationships of value types, despite the scores in his study being above 0.15 .

The normalized stress score for the espoused organizational values data was recorded as 0.1014 for a two-dimensional solution and so was well within the guideline figure for providing confidence in the resulting graphical display. PROXSCAL therefore generated a two-dimensional representation of the spatial relationships between values based upon the aggregated card sort. The dimensions are generated by the software and do not reflect any a priori interpretations: the relative placing of the value items indicates the best fit distance of each value to every other. The resulting array places all the value labels in a two-dimensional space, as shown in Fig. 1, such that distance equates to the perceived relationship of one value to another. The maximum distance between any two values is 1.0 , so the overall shape of the map is circular, with items at the perimeter having scores closer to 1.0 , revealing higher similarities with other values in the same area.

Small distances between value labels indicate a high degree of perceived similarity between the items, as for example in a cluster in the centre-right area that includes 'equality', 'diversity', 'democracy', 'inclusion', 'transparency' and 'life quality'. Conversely, comparatively greater distances between value labels indicate increasingly lower degrees of similarity. Overall, the 85 values in the inventory are distributed over the entire two-dimensional space fairly evenly; while there are some areas with more concentration of values than others, there are no obviously large empty spaces.

\section{Discussion}

This study set out to generate an inventory and conceptual map of espoused organizational values. This was created from those that organizations, across different sectors and in the UK and USA, espouse on their websites. It therefore represents a contemporary lexicon of values from a range of organizations. An initial list of values items was reduced to an inventory of 85 value labels; these were then arranged into groups of similar meanings via a card sort exercise. The resulting similarity matrix was in turn subjected to multidimensional scaling to generate a graphical display representing the relative relationship of each of the identified value labels in spatial terms, from which broad dimensions have been identified in a conceptual map. This map represents the first example of an empirically derived structure of espoused organizational values drawn from the terms being applied in organizations. 


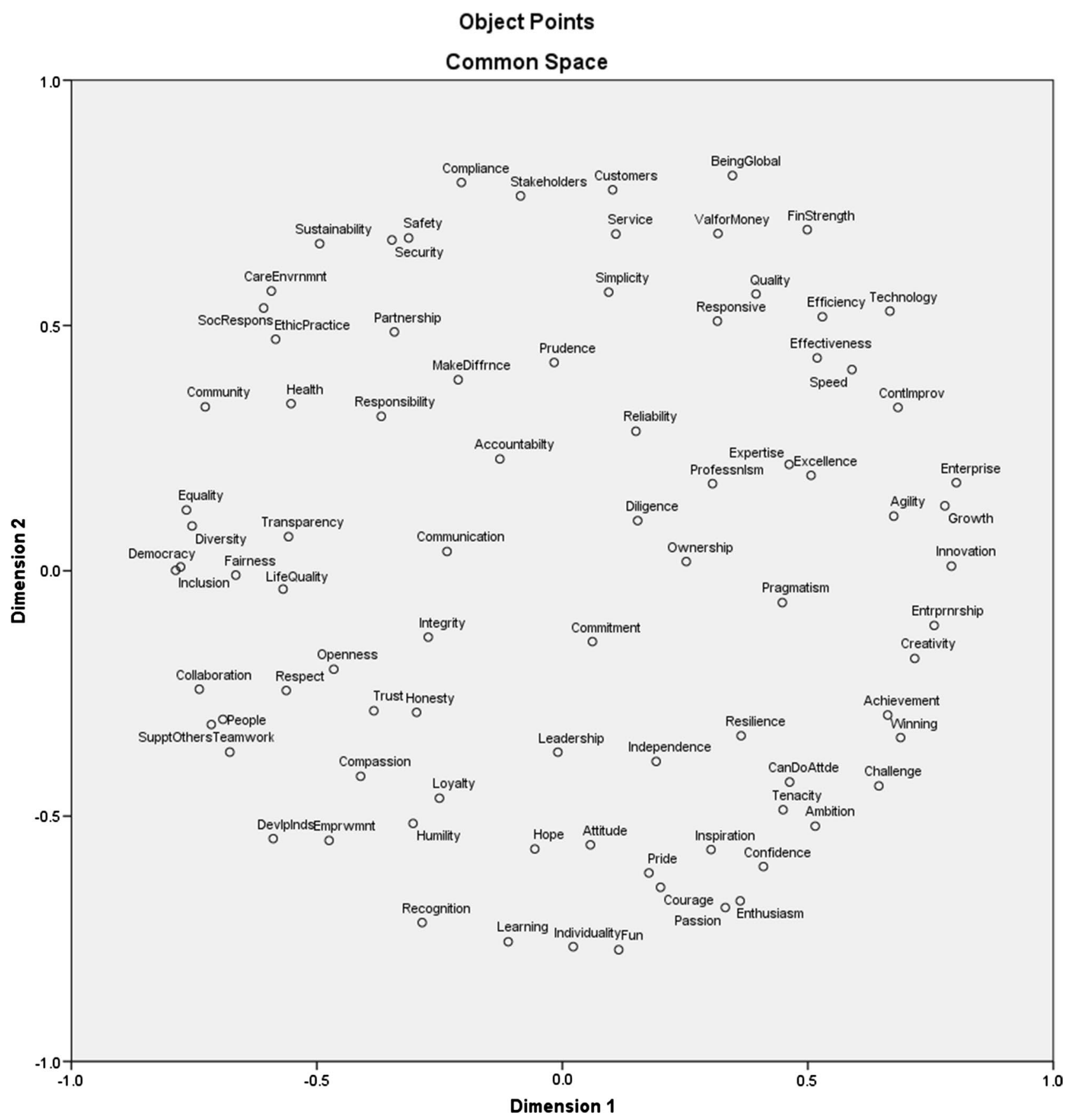

Fig. 1 PROXSCAL graphical display of espoused organizational values in a two-dimensional space

The study raises a number of points. First, the study focuses on espoused values, which, as Bourne and Jenkins (2013) point out, are an important and distinctive category of the values found in organizations. Espoused values are the most visible and accessible form of organizational values, and are frequently taken to represent the organization's values (e.g. Bansal 2003; Kabanoff et al. 1995; Voss et al. 2000). In carrying out this study, we found that $74 \%$ of UK and $80 \%$ of US organizations place statements of espoused values on their websites. The proportion has risen over the past two decades compared to the less than 10\% that McDonald and Gandz (1991) found when compiling their list of business values some 25 years earlier. Regardless of their proximity to the 'reality' of organizational life, espoused values are an important category of organizational values, and many questions regarding the patterns of espoused values by sector, industry and nation remain hitherto unexplored. 
In this discussion section, we first reflect on the nature of the conceptual map and make tentative suggestions as to the relationships and distinctions between values labels. We then compare the values found in our study of espoused values to those values in previous values relating to values more broadly. Finally, we consider the implications of our study for ethical practice within organizations.

\section{Reflections on the Conceptual Map of Espoused Organizational Values}

We can gain insights into espoused values by examining the conceptual map closely and reflecting on the potential relationships between values. Across the map there are instances where items are located close together. For example, the value labels of 'democracy' and 'inclusion', of 'equality' and 'diversity' and of 'people' and 'supporting others', all in the centre-right area of the display, occupy very similar spaces on the map. This indicates that in the card sort exercise, respondents considered them to be closely associated with one another. There are other items that are quite isolated, such as the value label 'commitment', which occupies a space near to the centre of the map and is at a greater distance from other value labels. An item located close to the centre would indicate a relationship with a number of other labels spread across the space. In the case of 'commitment', it is reasonable to suggest that this is a value that could be associated with many others as it represents a mode of action rather than an end-state (Rokeach 1973). Others located relatively centrally than others include 'communication', 'accountability' and the value label, 'integrity', each of which can be associated with many other labels without suggesting conflict or distance. Conversely, value labels located in close proximity towards the perimeter of the map, such as 'supporting others', 'teamwork', 'collaboration' and 'people', were frequently allocated to the same card sort groupings. Their proximity and location at the perimeter indicates that the values are closely associated with one another. Clustering of value labels that have similar sentiments to one another is apparent in a number of areas on the map. For example, there is a cluster at the centre-left edge with labels including 'equality', 'diversity', 'transparency', 'inclusion' 'life quality' and 'fairness', another towards the top-left including 'care for the environment', 'social responsibility' and 'ethical practice' and another in the bottom-right around the values of 'tenacity', 'ambition' and 'can-do attitude'.

The location of value labels in relation to one another reveals important associations. The value labels clustering around equality and fairness on the centre-left edge are located at greatest distance on the graphical display from another cluster comprising the values 'enterprise', 'growth', 'innovation', 'enterprise' and 'creativity' at the centre-right edge. The divergence between these clusters indicates that, in the context of organizations, these espoused values are perceived as occupying distinctly different spaces within the aggregated mental models of the card sort respondents. Likewise, a grouping of value labels including 'pride', 'passion', 'courage' and 'enthusiasm' towards the bottom-right of the display are seen as similar to one another but not similar to 'safety' or 'security' at the top-left. The association between value labels decreases the further one travels around the edge of the graphical display in either direction. On the left-hand side, for example, the values clustering around diversity and equality are quite close to those above, clustering around social responsibility, and those below, clustering around collaboration and teamwork. They are more distant, however, from values clustering around pride and passion in the lower-right area and from efficiency and financial strength in the top-right. In Schwartz's (1992) interpretation of personal values, the further one travels around the model from proximate values, to orthogonal and then opposite values, the greater the potential for values conflict or incompatibility. However, because of the nature of organizations, seemingly disparate values are commonly accommodated even though Quinn and Rohrbaugh (1983) describe them as competing and so give rise to some degree of tension. One important aspect of espoused values is that they are presented as a collective, and therefore, unlike individual values (Schwartz 1992) may need to coexist even if they have the potential to create tension.

The pattern of clustering of espoused value labels, as one travels around the perimeter of the map, suggests four distinct areas: an emphasis on community; an emphasis on competence; an emphasis on character and an emphasis on the interpersonal. Values in the upper-left quarter of the map broadly emphasize community concerns including sustainability, social responsibility, partnership and community itself. This area encompasses values that, in the aggregated view of the card sort respondents, include notions of responsibility, safety and health, and adds up to a sense of 'the other' in the broad dichotomy of 'the self' versus 'the other'. Values in the upper-right quarter, by contrast, emphasizes competence in carrying out the work of the organization-in outputs such as financial strength and growth, and in modes of action including efficiency, effectiveness and value-formoney. In the lower-left corner, values emphasize interpersonal relationships within organizations, including teamwork, trust, respect and honesty. Finally, in the area to the lower-right, there is a shift towards values concerned more about the character adopted by members in their workincluding ambition, resilience, confidence and enthusiasm. This shift could be taken to imply that there is an area in the map that has more 'instrumental' values (the way we do it) than terminal ones (what we seek to achieve). Rokeach (1973) separated instrumental and terminal values in his inventory, as did Schwartz and Bilsky (1987) in their first 
exploratory work. Later, however, Schwartz (1992) tested for this more comprehensively, concluding that there was 'little support for the idea that the terminal-instrumental distinction is a meaningful basis on which people organize their values' (Schwartz 1992, p. 37). Our map of espoused values suggests that different foci of values do exist, but does not support a clear separation between these two types.

Further to these areas of emphasis, it is notable that the espoused values in the upper half of the map tend to be outward facing, while those in the lower half tend to be inward facing, a dichotomy that is similar to Quinn and Rohrbaugh's (1983) 'external concerns' versus 'internal concerns' dimension. Likewise, values to the right-hand half tend to focus on task attributes-what is done and how-while those in the left-hand half tend to focus on ethical attributes-issues of morality and concern for others represented through values ranging from compassion, trust and supporting others, through equality and fairness to those concerned with community including sustainability and responsibility.

We can therefore suggest a number of areas of emphasis in our map of espoused values as depicted in Fig. 2.

\section{Comparison to Extant Frameworks of Organizational Values}

As the study captures the words used by organizations in their espoused values statements, the terms described as 'values' frequently extend beyond a precise academic definition of a value, as distinguished from an attitude, goal or principle (Rokeach 1968). Organizations often express their values using terms that do not closely match those found in academic lists of values (such as those created by McDonald and Gandz 1991; Rokeach 1973; or Schwartz 1992). In addition, scholars have commented on the lack of consistency in the academic literature regarding the distinction between values, beliefs and attitudes. For example, Kluckhohn (1951, p. 412) refers to 'confusion in discussion about values' because speakers had different categories in mind, while Smith (1969, p. 97) is "embarrassed by the proliferation of concepts akin to values' and Rohan (2000, p. 255, original italics) points out that people 'use the word values ... [to] mean just what they choose it to mean'. Given the variation within the academic literature concerning what constitutes a value, it is no surprise that the words and descriptions used by organizations also blur lines between values and more specific forms of belief. The value label 'customers', for example, may not conform to an academic definition of a value, but is espoused by a great number of organizations. When turned into the phrase 'we value our customers' it is evident that beneath the label there is a sentiment consistent with the concept of values, in this case outward facing concern for a specific group with whom the organization has close contact. Our intention in deriving an inventory based on the terms in use by organizations is to capture values as they are actually espoused and to therefore provide a consistent basis for future comparative and longitudinal work in this area.

In Table 1 we compare our inventory of espoused values and the resulting areas of emphasis with some of the prior lists and frameworks. In the first column, we arrange our inventory of values under each of the four areas of emphasis suggested above and then array other lists against these, ordering them in such a way to best retain their integrity while broadly matching ours. To help with clarity, we have spaced the lists so that values are aligned across the rows.

A first observation is that our inventory of espoused values has similarities with previous frameworks on organizational values in general. For example, all include values that are concerned with capability, including performance, efficiency, flexibility and adaptability. Similarly, all include some values that relate directly to character and to the interpersonal. However, there are categories in our inventory that are not evident in most of the prior frameworks. In particular, it is notable that values that reside in the 'Emphasis on Community' area on the conceptual map are almost wholly absent from other lists of values, value labels such as 'sustainability', 'care for environment', 'social responsibility' and 'ethical practice'. There are a number of reasons for why this might be the case. It may be, for example, that such values have only recently become sufficiently prominent in societies to be incorporated into organizations' espoused values. Alternatively, their omission from earlier frameworks may reflect the methods by which the value items in other studies were generated. We have observed that the focus of Kabanoff et al.'s (1995) framework is internal so might not be expected to include such externally facing values. Those that are derived through interview (e.g. McDonald and Gandz 1991) may reflect the scope that informants place on their concept of organizational values, while those that draw from previous literatures (e.g. Kalliath et al. 1999; O'Reilly et al. 1991) may simply repeat omissions made by earlier researchers. A further, more important, explanation may be that espoused values include categories that do not emerge from studies that focus on individual members' perspectives on their organizational values, such as the attributed and aspirational values identified by Bourne and Jenkins (2013).

Other lists also do not include values such as 'achievement', 'winning' and 'challenge'-their presence as espoused values may capture senior managers' desire for competitiveness within their chosen markets. The emergence of this category may be specific to espoused values as these labels relate strongly to the goals and objectives of organizations. There are also areas in the prior frameworks which have not been evident in our analysis of espoused values. These relate to 'authority' and 'reward' (Kabanoff et al. 1995), and to 'obedience' (McDonald and Gandz 1991). At first glance 


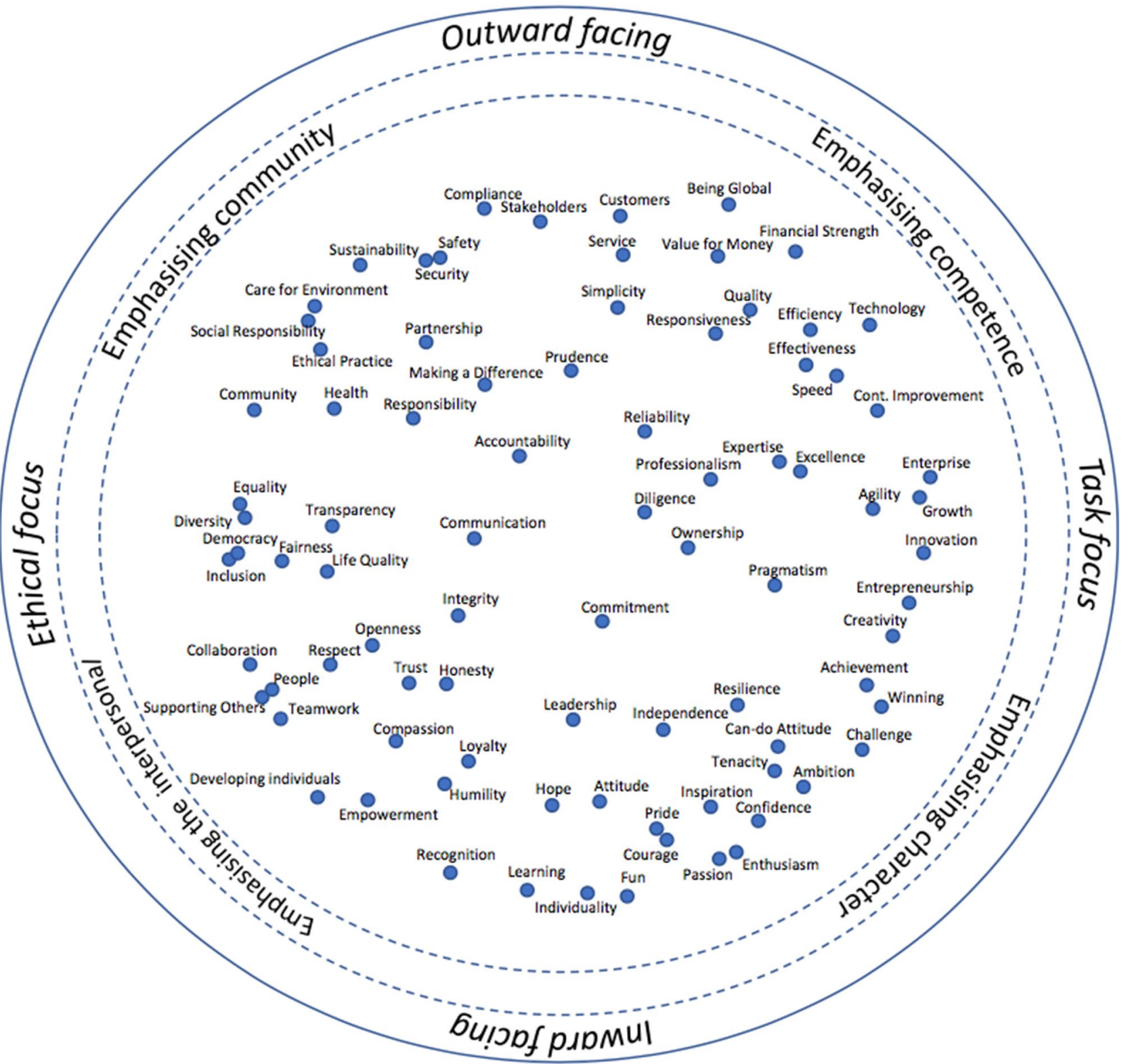

Fig. 2 A conceptual map of espoused organizational values

these suggest that in articulating espoused values externally, organizations avoid particular categories that they may consider to be contentious or even subversive to one or other of their key stakeholder groups. Employees may appreciate values emphasizing reward but may be less enthusiastic about those that imply control, while shareholders may appreciate authority values but be less enthusiastic about an emphasis on reward. As public statements, espoused values serve to influence stakeholders, in particular those who currently work or invest in the organization or who might do so in the future. Kabanoff and Daly's (2002) recognition that espoused values reflect the aspirations that senior managers have, and would like stakeholders to believe about their organizations, suggests that only the socially desirable ones are espoused while others are not, and for this reason, certain values that members attribute to their organization may not ever appear as espoused values. In commenting on the absence of value items relating to social power and influence in Rokeach's (1973) inventory of personal values, Schwartz and Bilsky (1987) noted that they were left out because Rokeach simply did not believe anyone would confess to them. The absence of value labels that imply control, power or even subversion in any of the inventories, including ours, would indicate that this sentiment applies to espoused as well as personal values but for different reasons. A further reflection on these issues is that they are less about omitting particular categories of 


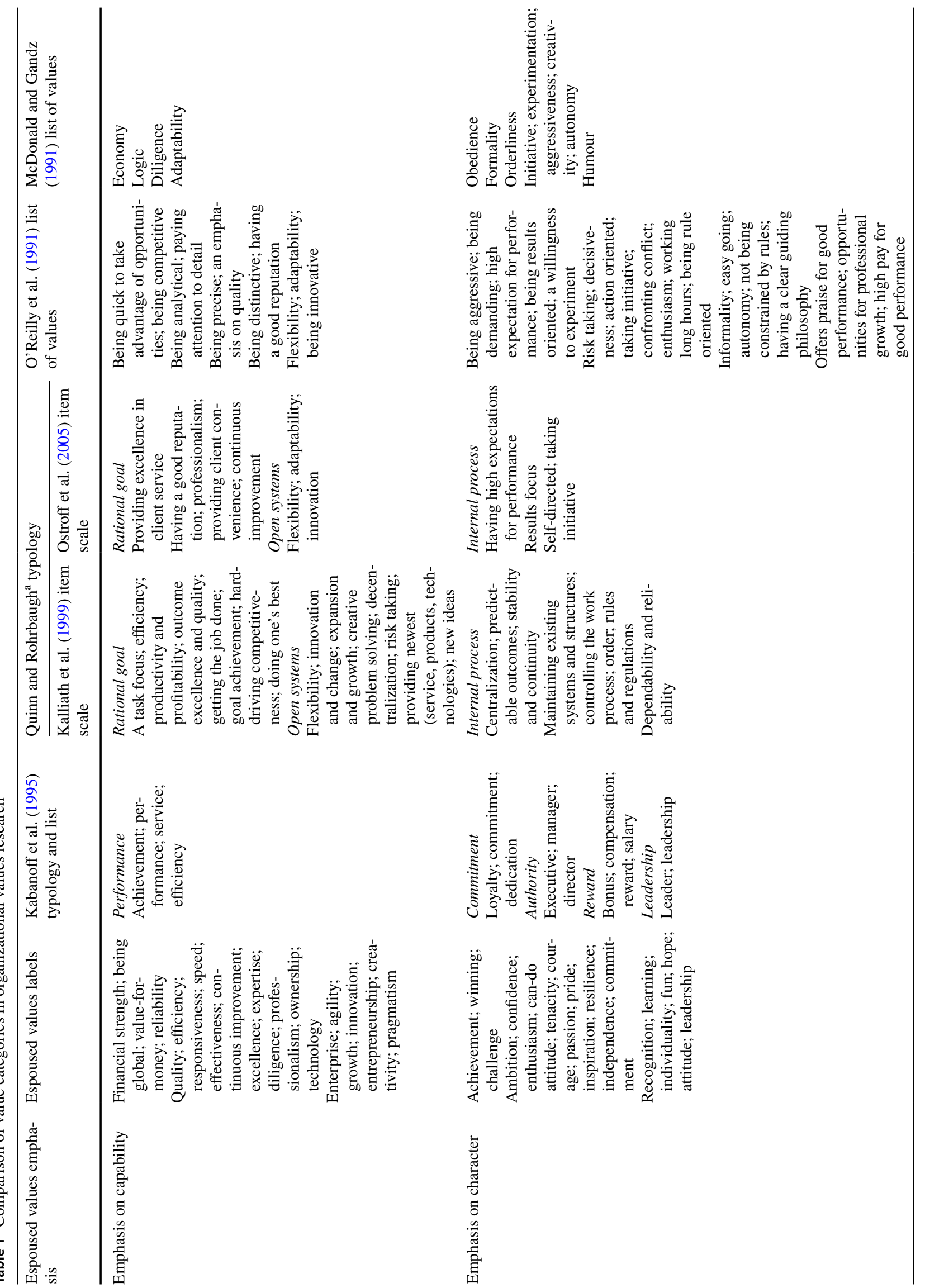




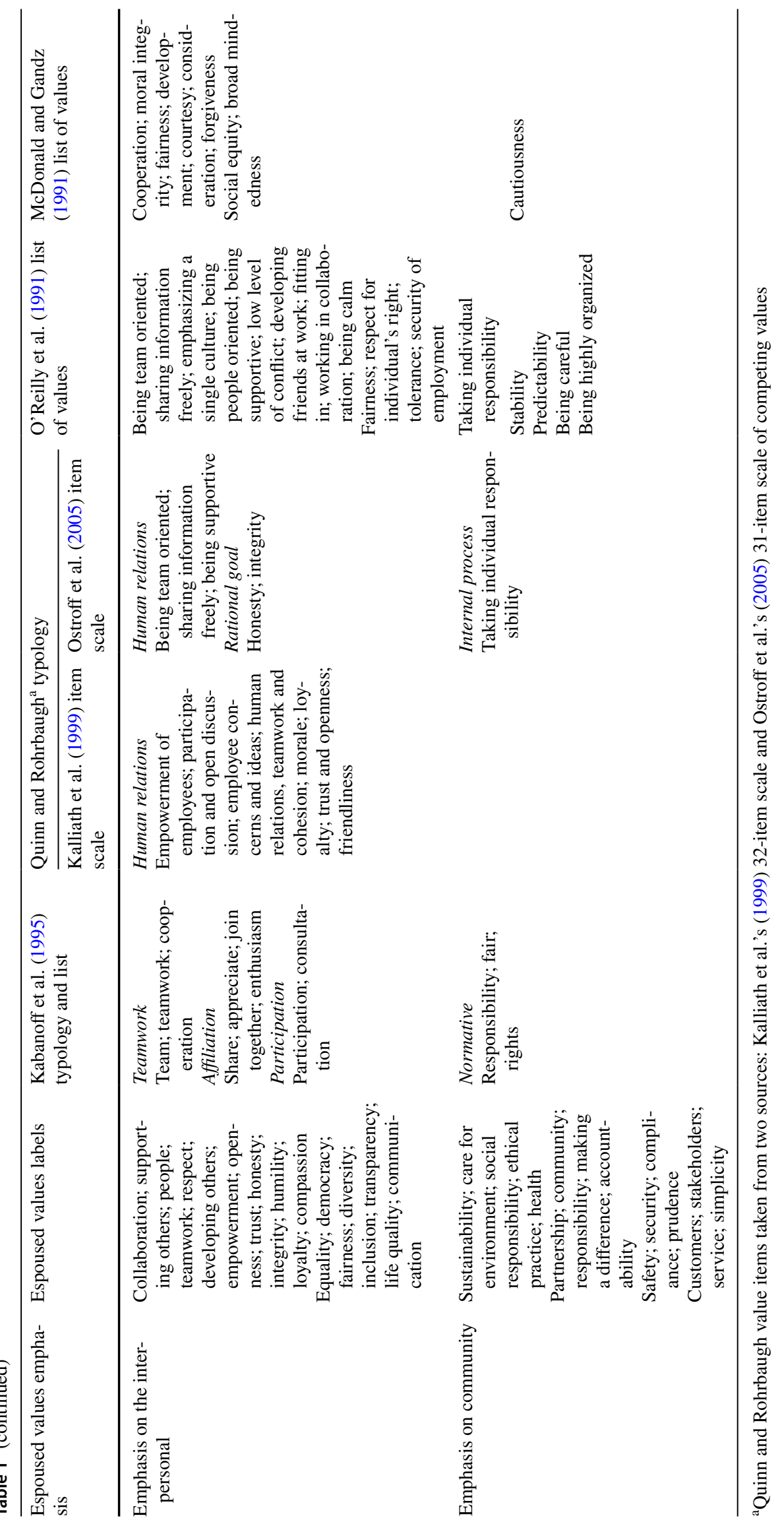


values, but more about reframing these into a language that is more acceptable to the various stakeholders. For example, authority could be re-positioned as leadership; reward as recognition and obedience as compliance. In these cases, there is evidence that some of the underlying value items remain, but that the language and terminology of espoused values is directed in a way which makes them broadly acceptable to a wide range of stakeholders.

\section{Implications for Ethical Practice Within Organizations}

So what does this suggest for ethical practice within organizations? If we use the earlier example of Enron, the implication is that espoused values have no relationship to ethical practices in the organization. However, over the last 25 years we have seen a significant growth in the adoption of espoused values by organizations. Our analysis suggests a strong ethical focus is an important area of emphasis in espoused values. This can be seen in Fig. 2 where we can identify value labels with both an inward (e.g. developing individuals) and outward (e.g. care for the environment) orientation. So is this just a case of cynical impression management or is this phenomenon more complex? Our study suggests that espoused values, because they have to be presented as a collection of organizational values, are more likely to coexist than compete: we need to be agile and we need to be reliable; we need our people to be individuals and we need them to work as a team. In espoused values terms these are not 'either/or' but 'both/and'together in paradox. Whereas individual value inventories focus on prioritising competing values, espoused values are presenting a group of values that have to work together in order to provide sense and effectiveness in the organization. This notion of coexisting tensions aligns to the literature on organizational paradox, Smith and Lewis (2011) suggest that paradoxical tensions are an inevitable part of organizing and an aspect that organizations need to continually resolve in order to be sustainable. Furthermore, the very richness of value labels that relate to ethical practices in the organization may suggest an increasing sophistication in our recognition of ethical behaviours and how these may develop in organizations. For example, in a study of individual values of managers, Weber (2015) found greater evidence of managers emphasizing moral value orientations in the early 2010s than in an earlier study in 1990. The implication being that espoused values are not simply a function of the desire to appease stakeholders, but are also an evolution of the constructs used within the organization to recognize and practice behaviours that will have a wider, more beneficial impact on society as a whole. The notion that espoused values represent the tensions resulting from the paradox inherent in the organization and provide a mechanism for evolving our understanding and practice of ethical behaviours are tentative concepts that may enable further development in the way that we consider, elicit and assess espoused values.

We argued earlier that the development of an inventory of espoused values and the associated conceptual map offers a first step in building theory regarding organizational, as opposed to individual, values. Organizations invest time and money into developing their espoused values, but we know little about why and how the values they espouse have emerged. The conceptual map offers the basis for beginning to explore pertinent questions, such as: how do the espoused values of organizations vary from nation to nation, sector to sector and industry to industry? What explains any differences observed - are there institutional drivers for the values that organizations espouse? Do we observe a herd instinct, or are there organizations who buck the trend, and if so, how do they perform compared to the industry or sector as a whole? If the values espoused by individual organizations, industries or sectors are tracked over time, is it possible to identify trends, and if so can these be linked to patterns of change in the wider environment? We have noted, for example, that values relating to the environment and community are strongly evident in our inventory, but are absent from others. Might this be related to a concurrent emphasis on corporate social responsibility and to concerns about climate change and sustainability? And if so, what impact might alternative concerns in the global political arena have on values espoused in future?

Espoused values present a rich source of further work as they are accessible to researchers both because of their public nature and wide adoption. While there is work on organizational outcomes related to espoused values (e.g. Jonsen et al. 2015), the conceptual map offers a basis for expanding upon this and making comparisons within and across national, sector and industry boundaries. Similarly, the conceptual map and its inventory of value labels will allow the tracking of espoused value priorities over time through the analysis of historical organizational documents and related to significant events, including, for example, acquisition and merger or international expansion. A further extension of this work would be to connect it to the work on ethical issues and their relationship to codes of ethics-at present this work lacks a strong empirical basis for exploring differences across organizations (Kaptein and Schwartz 2008). A more specific focus on espoused values as the level of analysis for understanding such codes potentially provides a more rigorous and replicable basis for comparison. 


\section{Conclusions}

Understanding the espoused values of organizations is a relatively neglected area and one which has been inhibited by the lack of access to espoused value statements. We have begun the process of addressing the need for further understanding of this important topic by developing an inventory and conceptual map of espoused values drawn from organizational websites. This represents a first step in establishing a basis for further research in this area. In contrasting this with prior work on organizational values we are able to identify emergent values categories which have not been present in past studies; they may be specific to the particular characteristics of espoused values or due to the fact that many of the prior studies were conducted more than 20 years ago. Furthermore, we also find that our inventory did not include a number of previous categories. This again may be due to the nature of espoused values and the change in temporal context; however, we also suggest that we may also be seeing as a reframing of the labels of categories to make them more palatable to multiple stakeholders.

Our contribution is to the development of our understanding of espoused values; however, our focus on this one particular form of organizational value is also a limitation. Future research might take our inventory and use it to examine the values attributed to organizations by their members in order to surface such values, and find additional and hitherto undisclosed forms of organizational values. Further research may need to be undertaken, for example, by using qualitative and undirected questions to surface values that are not espoused but which are embedded in organizational structures and systems, perhaps we may call these 'hidden' values. In addition, our study is limited to using organizational websites as a means of identifying espoused values. We believe that this approach has allowed us to be relatively comprehensive in our inventory; however, it is possible that organizations might espouse their values in other locations such as internal documents and not include them on their websites. A further limitation to this study is that the inventory is developed from the espoused values of organizations based in the UK and the USA. Questions to be answered include the extent to which values differ across national and regional cultures, and what impact this may have on those in the inventory. A related point is that while the respondents who carried out the card sort exercise represented a mix of gender and nationality, they were located in the UK. A similar study but with a different profile of respondents located elsewhere may show some differences in their sorting of groups of values from which the relationships between values were derived.

These limitations aside, our inventory and conceptual map represent the important first steps in providing a more detailed understanding of espoused values. We have also been able to identify some of the more distinctive aspects of espoused values that set them apart from other values forms and which will aid theoretical development in this area. In particular, we suggest that espoused values can be seen as a representation of the tensions resulting from the paradox inherent in organizations and that they therefore provide a mechanism for evolving our understanding and practice of ethical behaviours. There is still much to do in order to understand organizational values and their relationship to organizational behaviour, but in focusing specifically on the nature and characteristics of espoused values we believe that we have made some progress along this path.

\section{Compliance with Ethical Standards}

Conflict of interest The authors declare that they have no conflict of interest.

Ethical Approval All procedures performed in studies involving human participants were in accordance with the ethical standards of the institutional and/or national research committee and with the 1964 Helsinki declaration and its later amendments or comparable ethical standards. This article does not contain any studies with animals performed by any of the authors.

Informed Consent Informed consent was obtained from all individual participants included in the study.

Open Access This article is distributed under the terms of the Creative Commons Attribution 4.0 International License (http://creativecommons.org/licenses/by/4.0/), which permits unrestricted use, distribution, and reproduction in any medium, provided you give appropriate credit to the original author(s) and the source, provide a link to the Creative Commons license, and indicate if changes were made.

\section{References}

Amis, J., Slack, T., \& Hinings, C. R. (2002). Values and organizational change. The Journal of Applied Behavioral Science, 38(4), 436-465.

Auster, E. R., \& Freeman, R. E. (2013). Values and poetic organizations: Beyond value fit toward values through conversation. Journal of Business Ethics, 113, 1-11.

Bansal, P. (2003). From issues to actions: The importance of individual concerns and organizational values in responding to natural environmental issues. Organization Science, 14(5), 510-527.

Borg, I., \& Groenen, P. (2005). Modern multidimensional scaling: Theory and applications. New York: Springer.

Bourne, H., \& Jenkins, M. (2013). Organizational values: A dynamic perspective. Organization Studies, 34(4), 495-514.

Buenger, V., Daft, R. L., Conlon, E. J., \& Austin, J. (1996). Competing values in organizations: Contextual influences and structural consequences. Organization Science, 7(5), 557-576.

Burnes, B., \& Jackson, P. (2011). Success and failure in organizational change: An exploration of the role of values. Journal of Change Management, 11(2), 133-162. 
Cable, D. M., \& Edwards, J. R. (2004). Complementary and supplementary fit: A theoretical and empirical integration. Journal of Applied Psychology, 89(5), 822-834.

Caesar's Entertainment. (2014). Caesar's code of commitment. Retrieved from www.caesarscorporate.com. Accessed $20 \mathrm{Feb}$ 2015.

Carlisle, Y., \& Baden-Fuller, C. (2004). Re-applying beliefs: An analysis of change in the oil industry. Organization Studies, 25(6), 987-1019.

Cha, S. E., \& Edmondson, A. C. (2006). When values backfire: Leadership, attribution, and disenchantment in a values-driven organization. Leadership Quarterly, 17, 57-78.

Charity Commission for England and Wales. (2013). Top charities: October 13. Retrieved from www.gov.uk/government/organizations/charity-commission. Accessed 13 Oct 2013.

Crane, A. (1999). Are you ethical? Please tick yes or no: On researching ethics in business organizations. Journal of Business Ethics, 20(3), 237-248.

d'Andrade, R. (2008). A study of personal and cultural values. New York: Palgrave MacMillan.

Fincher, S., \& Tenenberg, J. (2005). Making sense of card sorting data. Expert Systems, 22(3), 89-93.

Finegan, J. E. (2000). The impact of person and organizational values on organizational commitment. Journal of Occupational and Organizational Psychology, 73(2), 149-169.

Fischer, R., Vauclair, C.-M., Fontaine, J. R. J., \& Schwartz, S. (2010). Are individual-level and country-level value structures different? Testing Hofstede's legacy with the Schwartz value survey. Journal of Cross-Cultural Psychology, 41(20), 135-151.

Fortune 500. (2013). Fortune 500 2013. Retrieved from www.fortune. com. Accessed 12 March 2014.

Gagliardi, P. (1986). The creation and change of organizational cultures. Organization Studies, 7, 117-134.

Giguère, G. (2007). Collecting and analyzing data in multidimensional scaling experiments: A guide for psychologists using SPSS. Tutorials in Quantitative Methods for Psychology, 2(1), 26-37.

Gioia, D. A., Schultz, M., \& Corley, K. G. (2000). Organizational identity, image, and adaptive instability. Academy of Management Review, 25(1), 63-81.

Grøgaard, B., \& Colman, H. L. (2016). Interpretive frames as the organization's "mirror": From espoused values to social integration in MNEs. Management International Review, 56(2), 171-194.

Howell, A., Kirk-Brown, A., \& Cooper, B. K. (2012). Does congruence between espoused and enacted organizational values predict affective commitment in Australian organizations? The International Journal of Human Resource Management, 23(4), 731-747.

Huntsman Corporation. (2014). Huntsman Corporation mission and values. Retrieved from www.huntsman.com. Accessed 20 Feb 2015.

J Sainsbury plc. (2014). J Sainsbury corporate website. Retrieved from www.j-sainsbury.co.uk/about-us/our-values/. Accessed 20 Feb 2015.

Jonsen, K., Galunic, C., Weeks, J., \& Braga, T. (2015). Evaluating espoused values: Does articulating values pay off? European Management Journal, 33(5), 332-340.

Kabanoff, B., \& Daly, J. (2002). Espoused values of organizations. Australian Journal of Management, 27(1), 89-104.

Kabanoff, B., \& Nesbit, P. (1997). Metamorphic effects of power as reflected in espoused organizational values: Implications for corporate governance. Australian Psychologist, 32(1), 62-70.

Kabanoff, B., Waldersee, R., \& Cohen, M. (1995). Espoused values and organizational change themes. Academy of Management Journal, 38(4), 1075-1104.

Kalliath, T. J., Bluedorn, A. C., \& Strube, M. J. (1999). A test of value congruence effects. Journal of Organizational Behavior, 20, $1175-1198$.
Kaptein, M., \& Schwartz, M. S. (2008). The effectiveness of business codes: An examination of existing studies and the development of an integrated research model. Journal of Business Ethics, 77, 111-127.

Kluckhohn, C. (1951). Values and value-orientations in the theory of action: An exploration in definition and classification. In D. Lerner \& H. D. Lasswell (Eds.), Toward a general theory of action (pp. 388-433). Cambridge, MA: Harvard University Press.

Kristof, A. L. (1996). Person-organization fit: An integrative review of its conceptualizations, measurement, and implications. Personnel Psychology, 49(1), 1-49.

Kruskal, J. B. (1964). Nonmetric multidimensional scaling: A numerical method. Psychometrika, 29, 115-129.

Kunen, J. S. (2002). Enron's vision (and values) thing. New York: New York Times.

Lencioni, P. M. (2002). Make your values mean something. Harvard Business Review, 80(7), 113-117.

McDonald, P., \& Gandz, J. (1991). Identification of values relevant to business research. Human Resource Management, 30(2), 217-236.

Nohria, N., \& Ghoshal, S. (1994). Differentiated fit and shared values: Alternatives for managing headquarters-subsidiary relations. Strategic Management Journal, 15, 491-502.

O'Reilly, C. A., III, Chatman, J., \& Caldwell, D. F. (1991). People and organizational culture: A profile comparison approach to assessing person-organization fit. Academy of Management Journal, 34(3), 487-516.

Ostroff, C., Shin, Y., \& Kinicki, A. J. (2005). Multiple perspectives of congruence: Relationships between value congruence and employee attitudes. Journal of Organizational Behavior, 26(6), 591-623.

Pruzan, P. (2001). The question of organizational consciousness: Can organizations have values, virtues and visions? Journal of Business Ethics, 29, 271-284.

Quinn, R. E., \& Rohrbaugh, J. (1983). A spatial model of effectiveness criteria: Toward a competing values approach to organizational analysis. Management Science, 29(3), 363-377.

Randall, D. M., \& Fernandes, M. F. (1991). The social desirability response bias in ethics research. Journal of Business Ethics, $10(11), 805-817$

Rohan, M. J. (2000). A rose by any name? The values construct. Personality and Social Psychology Review, 4(3), 255-277.

Rokeach, M. (1968). Beliefs, attitudes, and values: A theory of organization and change. San Francisco, CA: Jossey-Bass.

Rokeach, M. (1973). The nature of human values. New York: The Free Press.

Rosenberg, S., \& Kim, M. P. (1975). The method of sorting as a data gathering procedure in multivariate research. Multivariate Behavioural Research, 10, 489-502.

Schein, E. H. (1985). Organizational culture and leadership. San Francisco: Jossey-Bass.

Scholtens, B., \& Dam, L. (2007). Cultural values and international differences in business ethics. Journal of Business Ethics, 75, 273-284.

Schroders. (2014). Annual report and accounts, 2013. Retrieved from www.shroders.com/annualreport/files/pdfs/schrodersar13 corporateresponsibility.pdf. Accessed $20 \mathrm{Feb} 2015$.

Schwartz, S. H. (1992). Universals in the content and structure of values: Theoretical advances and empirical tests in 20 countries. In M. P. Zanna (Ed.), Advances in experimental social psychology (pp. 1-65). London: Academic Press.

Schwartz, S. H., \& Bilsky, W. (1987). Toward a universal psychological structure of human values. Journal of Personality and Social Psychology, 53(3), 550-562.

Scott, S. G., \& Lane, V. R. (2000). A stakeholder approach to organizational identity. Academy of Management Review, 25(1), 3-62. 
Smith, M. B. (1969). Personal values in the study of lives. In M. B. Smith (Ed.), Social psychology and human values: Selected essays (pp. 97-116). Chicago, IL: Aldine Publishing.

Smith, W. K., \& Lewis, M. W. (2011). Toward a theory of paradox: A dynamic equilibrium model of organizing. Academy of Management Review, 36(2), 381-403.

Stadler, C., \& Hinterhuber, H. H. (2005). Shell, Siemens and DaimlerChrysler: Leading change in companies with strong values. Long Range Planning, 38(5), 467-484.

Stride, H., \& Higgs, M. (2013). An investigation into the relationship between values and commitment: A study of staff in the UK charity sector. Nonprofit and Voluntary Sector Quarterly, 43(3), 455-479.

Voss, G. B., Cable, D. M., \& Voss, Z. G. (2000). Linking organizational values to relationships with external constituents: A study of nonprofit professional theatres. Organization Science, 11(3), 330-347.
Weber, J. (2015). Identifying and assessing managerial value orientations: A cross-generational replication study of key organizational decision-maker's values. Journal of Business Ethics, 132, 493-504.

Whaley, A. L., \& Longoria, R. A. (2009). Preparing card sort data f or multidimensional scaling analysis in social psychological research: a methodological approach. Journal of Social Psychology, 149(1), 105-115.

Wiener, Y. (1988). Forms of value systems: A focus on organizational effectiveness and cultural change and maintenance. Academy of Management Review, 13(4), 534-545.

Zander, L., Jonsen, K., \& Mockaitis, A. I. (2016). Leveraging values in global organizations: Premises, paradoxes and progress. Management International Review, 56(2), 149-169. 